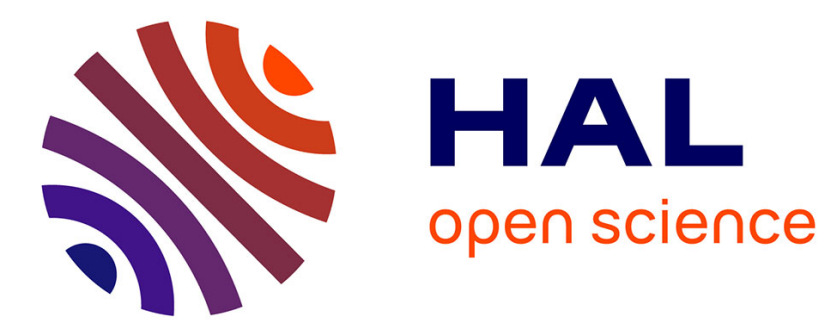

\title{
Compression à hautes températures et sous atmosphère oxydante de monocristaux de rutile $\mathrm{TiO} 2$
}

\author{
M. G. Blanchin, P. Faisant
}

\section{To cite this version:}

M. G. Blanchin, P. Faisant. Compression à hautes températures et sous atmosphère oxydante de monocristaux de rutile TiO2. Revue de Physique Appliquée, 1979, 14 (5), pp.619-627. 10.1051/rphysap:01979001405061900 . jpa-00244638

\section{HAL Id: jpa-00244638 https://hal.science/jpa-00244638}

Submitted on 1 Jan 1979

HAL is a multi-disciplinary open access archive for the deposit and dissemination of scientific research documents, whether they are published or not. The documents may come from teaching and research institutions in France or abroad, or from public or private research centers.
L'archive ouverte pluridisciplinaire HAL, est destinée au dépôt et à la diffusion de documents scientifiques de niveau recherche, publiés ou non, émanant des établissements d'enseignement et de recherche français ou étrangers, des laboratoires publics ou privés. 


\title{
Compression à hautes températures et sous atmosphère oxydante de monocristaux de rutile $\mathrm{TiO}_{2}(*)$
}

\author{
M. G. Blanchin et P. Faisant \\ Département de Physique des Matériaux (**), Université Claude-Bernard, 69621 Villeurbanne, France
}

(Reçu le 16 novembre 1978, révisé le 9 février 1979, accepté le 16 février 1979)

\begin{abstract}
Résumé. - Un appareillage pour effectuer des essais de compression à hautes températures (jusqu'à $1800 \mathrm{~K}$ ) sous balayage d'oxygène pur a été adapté sur une machine INSTRON. Ce montage permet de mesurer la déformation réelle de l'échantillon. Il a été utilisé pour déformer à vitesse constante des monocristaux stœchiométriques de rutile $\mathrm{TiO}_{2}$ entre 800 et $1700 \mathrm{~K}$. On a ainsi mis en évidence l'activation des systèmes de glissement $\{110\}\langle 001\rangle$ et $\{101\}<\overline{1} 01\rangle$ à partir de $900 \mathrm{~K}$. Les courbes contrainte-déformation relatives au second système peuvent être interprétées en termes d'interactions dislocations-défauts ponctuels extrinsèques.
\end{abstract}

\begin{abstract}
An apparatus for high temperatures (up to $1800 \mathrm{~K}$ ) compression tests under oxygen atmosphere has been built in an INSTRON machine. A special device is used for accurate measurements of the strain in the specimen. Constant strain rate deformation tests were performed on stoichiometric rutile $\mathrm{TiO}_{2}$ single crystals between 800 and $1700 \mathrm{~K}$. The two slip systems $\{110\}\langle 001\rangle$ and $\{101\}\langle\overline{1} 01\rangle$ were active above $900 \mathrm{~K}$. The stressstrain curves for this last system may be explained in terms of interaction between dislocations and extrinsic point defects.
\end{abstract}

1. Introduction. - Forme stable du dioxyde de titane $\mathrm{TiO}_{2}$, le rutile est actuellement l'objet d'un intérêt croissant en raison de ses différentes propriétés physiques [1], toutes fortement influencées par l'aptitude du rutile à perdre sa stœchiométrie sous pression partielle d'oxygène réduite. C'est pourquoi il est apparu nécessaire, dans un premier temps, d'obtenir des données expérimentales de référence quant à la déformation de monocristaux dont la stœchiométrie originelle est préservée au mieux par un balayage d'oxygène pur. La déformation plastique du rutile, comme celle d'autres oxydes métalliques, nécessite le développement de contraintes élevées à hautes températures $(>900 \mathrm{~K})$ qui, sous atmosphère oxydante contrôlée, rendent caduc l'emploi des dispositifs conventionnels utilisés pour la déformation des métaux.

Aussi présentons-nous ici un montage de déformation en compression pouvant fonctionner jusqu'à une température de $2073 \mathrm{~K}$ sous balayage d'oxygène pur à la pression atmosphérique. La mesure de la déformation réelle de l'échantillon est également accessible durant l'essai de déformation. Les solutions technologiques simples adoptées pour répondre à ces exigences permettent une réalisation rapide et de moindre coût. Enfin la conception du montage et son encombrement réduit en permettent l'adaptation

(*) Ce travail constitue une partie de la thèse de M. G. B., soutenue à Lyon en juillet 1978 .

(**) Associé au C.N.R.S. sur des machines d'essais de faibles dimensions (tel fut notre cas), comme sur des machines plus importantes en tant que partie d'un ensemble polyvalent.

Nous avons procédé grâce à ce montage à des essais de compression à vitesse constante sur des éprouvettes monocristallines massives de différentes orientations cristallographiques. Les résultats obtenus ont permis de mettre en évidence les systèmes de glissement principaux de $\mathrm{TiO}_{2}$ et de caractériser les propriétés mécaniques des cristaux dans les orientations correspondantes.

2. Dispositif expérimental. - 2.1 CARACTÉRISTIQUES GÉNÉRALES DE L'APPAREILLAGE. - L'usinage d'éprouvettes de traction étant rendu extrêmement difficile par la fragilité à basses températures, l'essai de compression uniaxiale est généralement utilisé [2] pour l'étude de la déformation des oxydes, ce mode de sollicitation étant d'ailleurs assez proche de ceux rencontrés lors d'applications industrielles [3]. Aux montages de compression indirects permettant théoriquement une meilleure conservation de l'axialité [4], nous avons préféré un montage direct dont trois avantages majeurs sont une meilleure rigidité, un encombrement réduit et une plus grande simplicité d'exécution (d'où un moindre coût) des pièces maîtresses.

La figure $1 a$ présente une vue générale du montage $\left({ }^{1}\right)$ adapté sur une machine d'essais INSTRON

(1) Une reproduction des plans originaux avec cotation peut être fournie au lecteur sur sa demande. 


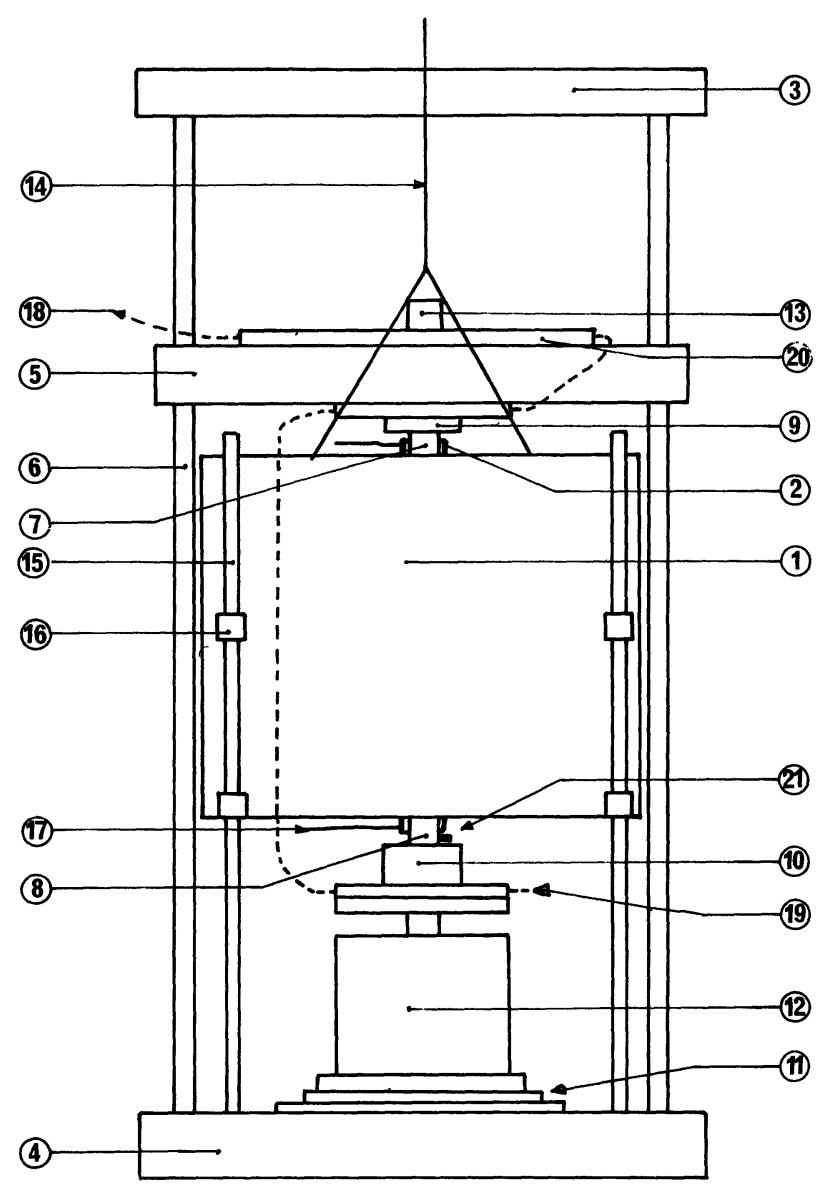

Fig. 1a. - Vue d'ensemble de l'appareillage adapté sur la machine d'essais.

[General view of the apparatus built in the testing machine.]

1 Four

2 Resistor

3 Traverse fixe

4 Table machine

5 Traverse mobile

6 Colonne de guidage de la traverse

7 Piston supérieur (alumine)

8 Piston inférieur (alumine)

9 Support piston supérieur

10 Support piston inférieur

11 Plaque pour les corrections de parallélisme et d'axialité

12 Cellule de compression

13 Capteur de déplacement

14 Câbles pour levage du four

15 Colonnes de guidage du four

16 Guides avec roulements à billes

17 Raccordement à l'alimentation du four

18 Alimentation en eau

TM-SM-L (capacité maximale $500 \mathrm{kp}$ ) de dimensions réduites. L'éprouvette (de hauteur $6 \mathrm{~mm}$ ) est comprimée entre deux pistons massifs dont l'un est solidaire de la traverse mobile et l'autre fixé au plateau de la cellule de compression. L'ensemble se trouve placé dans le résistor en chromite de lanthane (longueur $400 \mathrm{~mm}$, diamètre intérieur $42 \mathrm{~mm}$ ) d'un four PYROX 2000 (fabrication C.E.A.) permettant d'at-

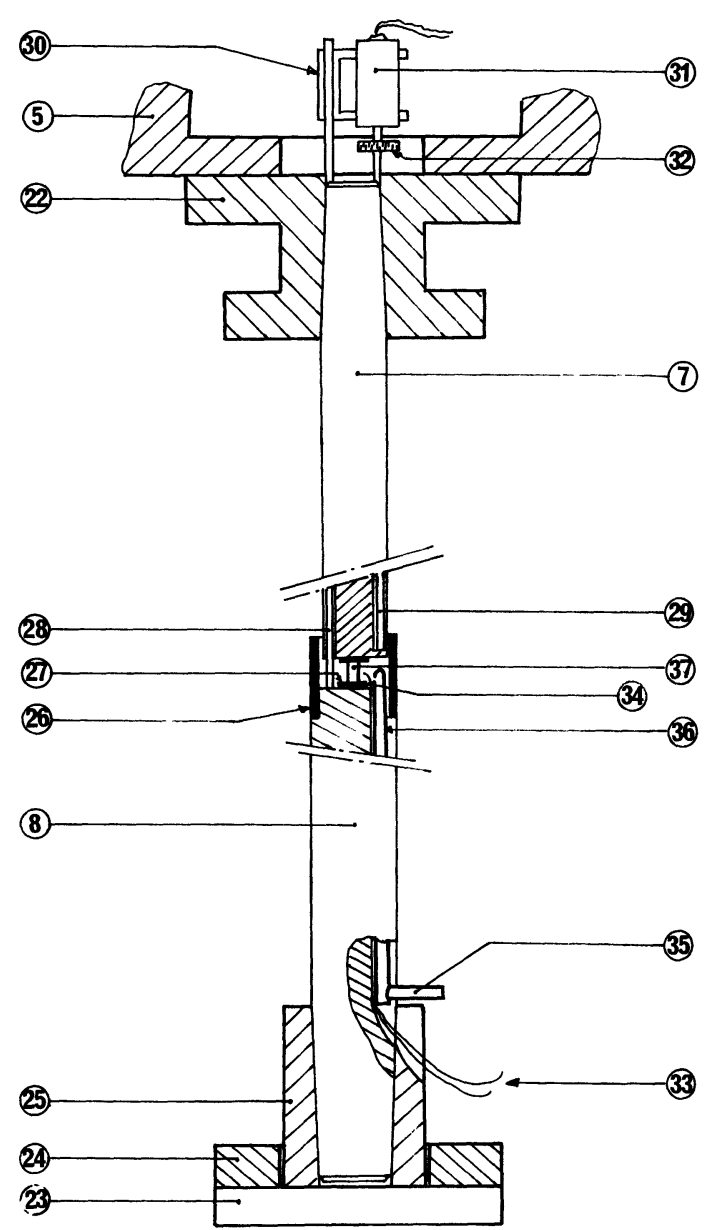

Fig. 1b. - Vue détaillée du montage de compression. [Details of the compression rams.]

19 Evacuation de l'eau

20 Plaque de refroidissement supérieure

21 Arrivée de l'oxygène

22 Support de piston supérieur (acier inoxydable)

23 Plateau cellule de compression (acier inoxydable)

24 Socle de refroidissement inférieur (acier inoxydable)

25 Support de piston inférieur (acier inoxydable)

26 Manchon (alumine)

27 Plaquettes (alumine)

28 Tige inférieure pour la mesure des déplacements (alumine)

29 Tige supérieure pour la mesure des déplacements (alumine)

30 Support du bobinage inducteur (dural)

31 Bobinage inducteur du capteur de déplacement

32 Positionnement du noyau du capteur

33 Thermocouples (régulation et mesure) : soudure froide

34 Thermocouples (régulation et mesure) : soudure chaude

35 Tube de raccordement (pour l'arrivée de l' $_{2}$ )

36 Tube pour insufflation de $\mathrm{l}^{\prime} \mathrm{O}_{2}$ (alumine)

37 Echantillon.

teindre des températures voisines de $2073 \mathrm{~K}$ sous oxygène pur [5]. Durant l'essai de déformation, le four, par l'intermédiaire d'un bâti métallique solidaire de sa carcasse isolante, repose sur la table fixe de la machine, de telle sorte que l'éprouvette se trouve au centre de la zone chauffante (longueur $60 \mathrm{~mm}$ ) du résistor. Le bâti peut coulisser vers le haut le long de glissières pour introduire l'échantillon. 
2.2 APPLICATION DE L'EFFORT DE COMPRESSION. Une vue détaillée de l'ensemble permettant la transmission de l'effort de compression a été représentée sur la figure $1 b$.

Les pièces maîtresses sont deux pistons cylindriques massifs (diamètre du piston inférieur à $36 \mathrm{~mm}$ ) en alumine polycristalline de haute densité (type DESMARQUEST AF 997) dont la résistance mécanique en compression reste très élevée jusqu'à $1800 \mathrm{~K}$. Les pistons sont ajustés dans les pièces métalliques correspondantes par emboîtements coniques (Fig. 1b) : ce mode de fixation confère au montage, après compression à vide, une rigidité maximale.

L'axialité de l'ensemble est réglée $a$ priori à température ambiante, grâce à la plaque de fixation 11 (Fig. 1a) qui possède trois degrés de liberté : deux translations ménagées par trois brides de fixation et une inclinaison variable par l'utilisation de cales. L'alignement des axes des pistons est effectué par circulation d'un gabarit cylindrique et le réglage du parallélisme entre les surfaces libres par déplacement d'une cale rectifiée. Les différents réglages effectués, la plaque 11 est totalement immobilisée par serrage et le défaut maximal de parallélisme obtenu est alors inférieur à $10 \mu$ sur un diamètre de $30 \mathrm{~mm}$. L'étude de la forme et des systèmes de glissement des échantillons, détaillée plus avant, montre que l'axialité ainsi acquise à température ambiante se conserve de manière satisfaisante à hautes températures.

2.3 MESURE DES TEMPÉRATURES SOUS ATMOSPHÈRE OXYDANTE. - La pression partielle d'oxygène de 1 atmosphère, nécessaire au maintien de la stœchiométrie de nos échantillons est obtenue grâce à un balayage d'oxygène extra-pur $\mathbf{N} 48$. Le gaz insufflé par la fente d'un tube en alumine haute densité jusqu'à quelques $\mathrm{mm}$ de l'éprouvette est confiné à l'entour par un manchon cylindrique en alumine (Fig. 1b), qui protège également l'échantillon contre toute évaporation d'impuretés à hautes températures en provenance $\mathrm{du}$ résistor $\mathrm{du}$ four (chrome en particulier). La pression d'insufflation (1,3 bar à l'entrée du tube) et le débit $(30 \mathrm{l} / \mathrm{h})$ ont été déterminés au cours d'essais préliminaires [6], durant lesquels on a vérifié que la pression partielle d'oxygène au niveau de l'échantillon demeure toujours très légèrement supérieure à 1 atmosphère.

On dispose de deux thermocouples logés entre le piston inférieur et le tube d'oxygène : un couple $\mathrm{Pt}$ $\mathrm{Rh} 10 \%-\mathrm{Pt} \mathrm{Rh} 40 \%$ relié à la régulation EUROTHERM (laquelle autorise un écart maximal de $\pm 0,1^{\circ}$ ) et un couple Pt-Pt $\mathrm{Rh} 10 \%$ pour la mesure proprement dite, sur laquelle l'erreur absolue a été estimée à $\pm 3^{\circ}$. La température de chaque essai, donnée par ce dernier couple, est mesurée à mihauteur de l'éprouvette. L'enregistrement du gradient thermique a montré que l'écart en température sur la longueur de l'éprouvette ne dépasse pas $6^{\circ}$ pour une température nominale de $1173 \mathrm{~K}$ mesurée à l'équilibre thermique.
2.4 MESURE DE LA DÉFORMATION RÉELLE DE L'ÉCHANTILLON. - Au cours d'essais préliminaires à différentes températures, où nous avons enregistré la déformation propre de l'appareillage lors de mises en charge effectuées piston contre piston, la déformation élastique de la machine INSTRON s'est révélée non négligeable (de l'ordre de $0,7 \mu / \mathrm{kp}$ à $1300 \mathrm{~K}$ ) [1]. C'est pourquoi il nous a paru nécessaire de mesurer la déformation réelle de l'échantillon au niveau des faces de compression à l'aide de deux tiges en alumine (Fig. 1b), dont le déplacement relatif est enregistré grâce à un capteur inductif PHILIPS PR 9301, associé à un pont d'extensométrie PHILIPS PR 9308 de haute précision.

L'étalonnage préalable de ce dispositif dans son ensemble a été effectué en déplaçant la traverse mobile à vitesse connue sans comprimer aucun cristal. L'incertitude relative sur les valeurs fournies par le système étalonné est de $3 \%$ [1].

3. Déformation de monocristaux de rutile. Ashbee et Smallman [7], [8] réalisèrent les premiers la déformation en compression uniaxiale de monocristaux synthétiques massifs de rutile, monocristaux présumés stœchiométriques. Cependant la pression partielle d'oxygène et la pureté de l'atmosphère gazeuse environnante n'étaient pas contrôlées au cours des essais. Or ces paramètres déterminent l'écart à la composition stœchiométrique idéale, susceptible de s'instaurer à hautes températures comme exposé ci-après. Dans le cas de la présente étude, au contraire, la pression partielle d'oxygène est fixée à 1 atmosphère, grâce à l'appareillage utilisé. Cette valeur nous permet d'évaluer assez précisément l'écart maximal à la stœchiométrie.

Nous avons utilisé des monocristaux synthétiques massifs non dopés, élaborés par méthode de Verneuil (fournisseur L.E.T.I., C.E.N.G.). Une analyse par spectroscopie d'émission optique (C.E.N. Saclay) a révélé un grand nombre d'impuretés, parmi lesquelles les espèces cationiques majoritaires sont, par ordre décroissant :

$$
\begin{aligned}
\mathrm{Al}(\simeq 200 \text { p.p.m. }), \mathrm{Si}(\simeq 100 \text { p.p.m. }) \\
\mathrm{Mg}(\simeq 40 \text { p.p.m. }), \mathrm{Fe}(\simeq 25 \text { p.p.m. }) .
\end{aligned}
$$

Après le processus de croissance proprement dit, les monocristaux subissent un recuit vers $1973 \mathrm{~K}$ sous débit d'oxygène pendant plusieurs heures, suivi d'un refroidissement lent, en 20 heures environ. A l'issue de ce traitement, on ne décèle pas d'écart à la composition stœchiométrique idéale qui soit mesurable par méthodes optique et électriques [1].

3.1 CONSERVATION DE LA STCECHIOMÉTRIE DURANT LA DÉFORMATION. - $\mathrm{TiO}_{2}$ représente l'oxyde de degré le plus élevé du système Ti-O. Tout écart à la composition stœchiométrique idéale $\mathrm{TiO}_{2}$ se traduit par une déficience en oxygène, d'où l'écriture habituelle du composé sous-stœchiométrique correspondant $\mathrm{TiO}_{2-x}, x$ dépendant à la fois de la température $T$ 
et de la pression partielle d'oxygène $p \mathrm{O}_{2}$. Pour des valeurs de $x<0,01$, de tels composés peuvent être analysés en termes de solutions solides de défauts ponctuels : interstitiels titane et lacunes d'oxygène, dans la matrice de structure rutile. Des études récentes $[9,10,11]$ consacrées à ce domaine de composition ont montré que le défaut ponctuel prédominant est l'interstitiel titane $\mathrm{Ti}_{\mathrm{i}}^{4+}$ pour les faibles valeurs de $p \mathrm{O}_{2}$.

Pour des valeurs de $p \mathrm{O}_{2}$ proches de $1 \mathrm{~atm}$ en revanche, la valeur plus élevée du coefficient de diffusion de l'oxygène [12] suggère un accroissement de la concentration des lacunes d'oxygène : on doit donc admettre, dans ces conditions, la coexistence des deux types de défauts ponctuels, interstitiels titane et lacunes d'oxygène, sans que l'on ait déterminé pour l'instant avec certitude lequel est prépondérant.

Dans un cristal idéalement pur, la concentration totale de tels défauts ponctuels serait extrêmement faible lorsque $p \mathrm{O}_{2}=1 \mathrm{~atm}$ : environ 1 p.p.m. à $1273 \mathrm{~K}$ [10], [11]. Dans un cristal impur en revanche, cette concentration est largement contrôlée, à certaines températures, par les impuretés cationiques en substitution dont la valence est différente de +4 . Dans nos cristaux, ces impuretés aliovalentes majoritaires sont $\mathrm{Al}^{+++}$et $\mathrm{Mg}^{++}$. Etant donnée leur concentration, nous avons estimé, en utilisant les constantes d'équilibre déterminées par Baumard [10], [11], la concentration des espèces $\mathrm{Ti}_{\mathrm{i}}^{4}$ et $\mathrm{V}_{0}^{2}$ en fonction de $T$ pour $p \mathrm{O}_{2}=1 \mathrm{~atm}$. La concentration de ces défauts apparaît constante et fixée par celle des impuretés jusqu'à $1600 \mathrm{~K}$ environ ; l'ordre de grandeur correspondant est de 70 p.p.m. si l'on suppose que l'interstitiel $\mathrm{Ti}_{i}^{4}$ reste prédominant, et de 140 p.p.m. si l'on admet au contraire la prédominance des lacunes $V_{0}^{2}$. Ces concentrations n'augmentent que légèrement pour des températures supérieures. Nous avons donc estimé que l'écart maximal à la stœchiométrie entre 800 et $1700 \mathrm{~K}$ (températures de nos essais) ne devait pas dépasser $1,4 \times 10^{-4}$, ce qui demeure très faible.

\subsection{Mise EN ÉvidenCE DE GLISSEMENTS ACTIFS. -} Le rutile cristallise dans le système quadratique, la maille élémentaire correspondante est reproduite sur la figure 2, le rapport $c / a$ valant 0,643 9. Ashbee et Smallman [7] ont recensé les systèmes de glissement actifs dans le rutile monocristallin non dopé. Ce serait :

- le système $\{101\}\langle\overline{1} 01\rangle$ (on compte quatre plans équivalents $\{101\}$ dans le réseau, avec une seule direction $\langle\overline{101}\rangle$ possible dans chaque plan),

- le système $\{110\}\langle 001\rangle$ (on compte deux plans équivalents $\{110\}$ comparant la même direction $\langle 001\rangle$, tous deux activables à partir de $875 \mathrm{~K}$ ).

3.2.1 Procédure expérimentale. - Dans les gros cristaux en provenance du fournisseur, nous avons donc découpé, à l'aide d'une scie à meule diamantée, deux séries d'éprouvettes parallélépipédiques de dimensions $2,8 \mathrm{~mm} \times 2,8 \mathrm{~mm} \times 6 \mathrm{~mm}$. Chaque série correspond à une orientation cristallographique

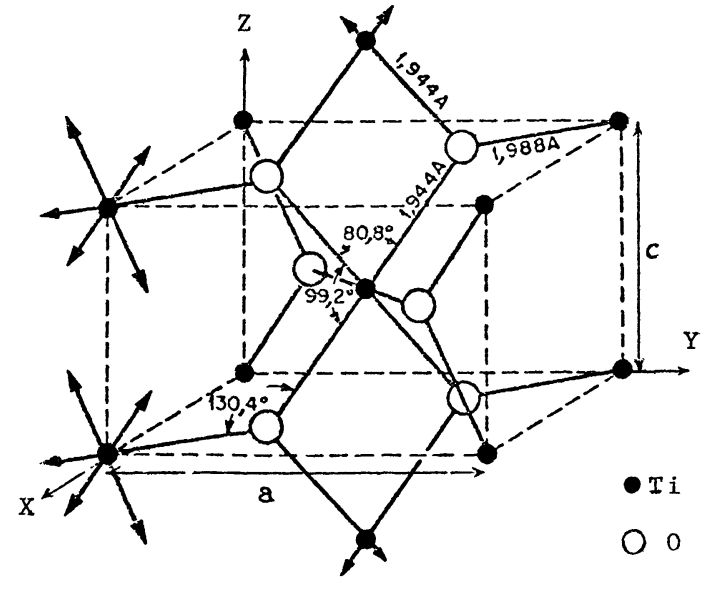

Fig. $2 a$. - Maille du rutile : $a=4,5937 \AA$ et $c=2,9581 \AA$. [Unit cell of rutile : $a=4.5937 \AA$ and $c=2.9581 \AA$.]
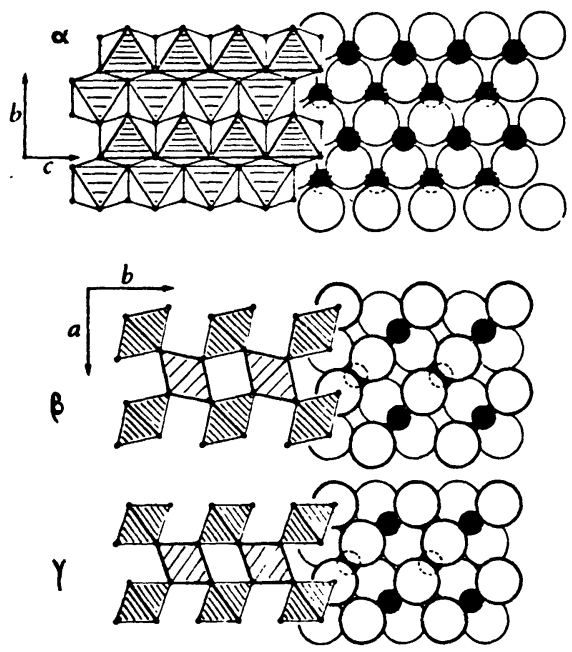

Fig. $2 b .-\alpha$ structure de rutile projetée parallèlement à l'axe [10]; $\beta$ structure du rutile projetée parallèlement à l'axe $[00] ; \gamma$ même projection qu'en $b$, mais dans la structure h.c.p. idéalisée (les plans (100) d'oxygène ne présentent plus d'ondulation).

choisie de sorte que l'effort de compression exercé parallèlement à la plus grande dimension des éprouvettes active préférentiellement l'un des deux systèmes de glissement, ainsi qu'il est exposé ci-après.

Nous présentons ici les résultats d'observations réalisées à l'échelle macroscopique : forme des échantillons et répartition des figures de corrosion sur les faces latérales de ceux-ci après déformation à vitesse constante.

Les figures de corrosion sont obtenues par immersion dans l'acide sulfurique bouillant après polissage chimique par fusion alcaline dans la potasse.

3.2.2 Activation des systèmes de glissement. a) Compression à $45^{\circ}$ de [001]. - Cette orientation des éprouvettes favorise au maximum la direction de glissement [001], située à $45^{\circ}$ de l'axe de compression dans le plan (010) (Fig. 3a). Deux plans de glissement (110) et (110) peuvent être activés admettant 


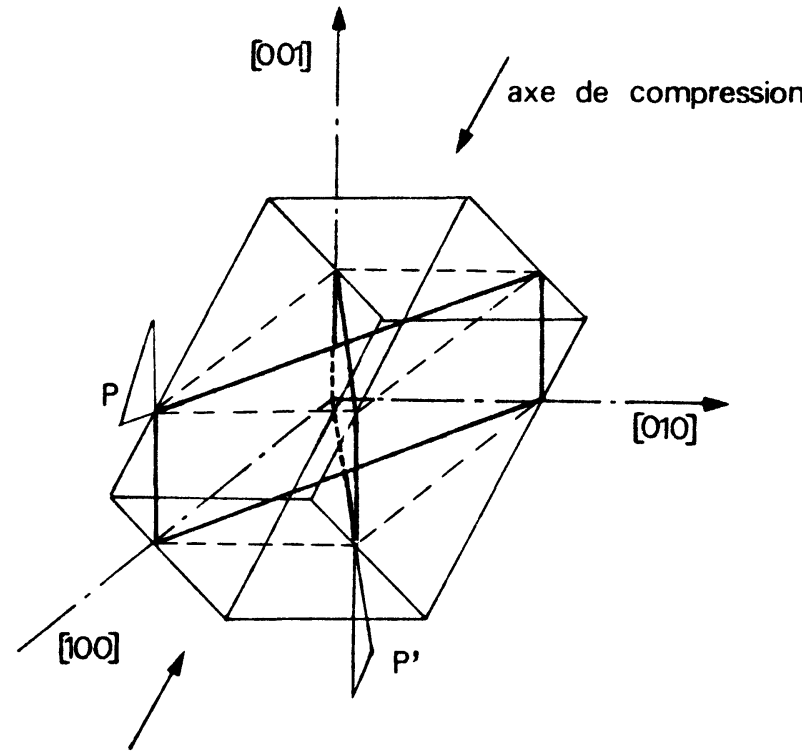

Fig. 3a. - Axe de compression à $45^{\circ}$ de [001] dans le plan (010). Les plans de glissement actifs sont $P$ et $\mathbf{P}^{\prime}$.

[Compression axis at $45^{\circ}$ with regard to [001] in the (010) plane. The active slip planes are $\mathbf{P}$ and $\mathbf{P}^{\prime}$.]

[001] comme direction de glissement commune. Les systèmes $\{101\}\langle\overline{1} 01\rangle$ sont également activables a priori quoique moins favorablement orientés.

Une attaque chimique, pratiquée après une déformation $\varepsilon \simeq 1,5 \times 10^{-2}$ obtenue par compression dans cette orientation, révèle une densité de figures de corrosion relativement faible sur les faces latérales des éprouvettes. Sur les faces (010) (Fig. 3b), on discerne cependant quelques alignements se propageant depuis la surface : leur direction à $45^{\circ}$ de l'axe de l'éprouvette correspond bien à la trace des

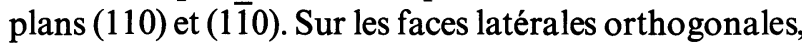
on ne décèle aucune trace de glissement, en particulier aucune susceptible de correspondre à l'activation des plans $\{101\}$.

b) Compression suivant l'axe [001]. - Pour cette seconde série d'éprouvettes, l'effort de compression est exercé parallèlement à la direction [001], qui est

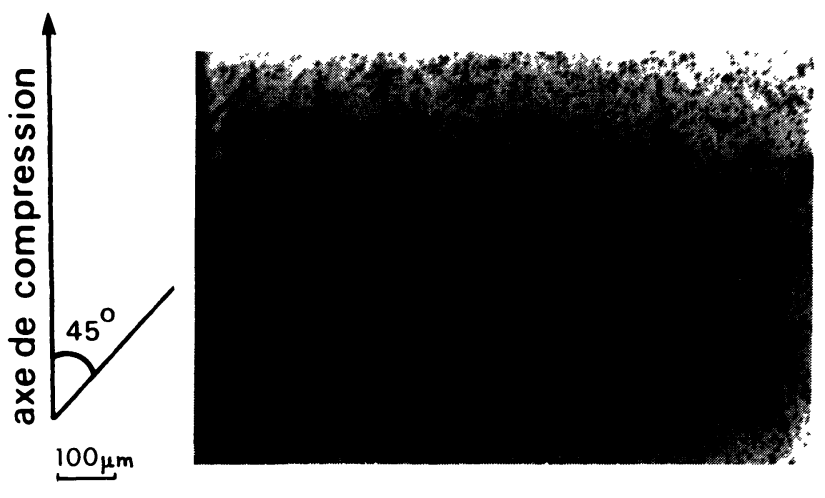

Fig. $3 b$. - Face latérale (010) d'une éprouvette déformée dans cette orientation.

[Lateral face (010) of a sample deformed in this orientation.] l'axe de symétrie d'ordre 4 (Fig. $4 a$ ). On voit que le système $\{110\}<001>$ ne peut être activé dans ces conditions. Parmi les autres systèmes de glissement simples envisageables a priori, le système $\{101\}$ $\langle\overline{1} 01\rangle$ possède en revanche le facteur de Schmid maximal $(A=0,455)$.

La symétrie d'ordre 4 implique donc l'existence d'un glissement quadruple sur les plans équivalents $\{101\}$, qui est confirmée par la configuration des figures de corrosion sur les faces latérales des éprouvettes comprimées dans cette orientation. Les clichés de la figure $4 b$, par exemple, ont été obtenus sur les faces d'une éprouvette déformée à $1068 \mathrm{~K}$, peu après la limite élastique. On constate que les figures de corrosion sont alignées suivant deux directions symétriques par rapport à [001] et faisant entre elles un angle compris entre 65,5 et $66^{\circ}$. Il s'agit donc bien des traces des plans $\{101\}$, l'angle entre (101) et (001) valant $32,8^{\circ}$. Des observations en microscopie électronique [13] ont, par ailleurs, mis en évidence les mêmes plans de glissement et permis de confirmer la direction de glissement $\langle\overline{1} 01\rangle$. A cette température, les quatre plans paraissent également activés et ce, de manière assez homogène tout au long de l'éprouvette. On remarque seulement au niveau de chaque tête la présence du cône de glissement d'axe [001], région où la densité des traces de glissement est moindre en raison de l'adhérence de l'extrémité du cristal au piston.

L'égale activation des quatre systèmes de glissement prouve la bonne axialité de l'effort de compression exercé. Des clichés macrographiques effectués sur des échantillons déformés à des taux croissants [1] montrent que la forme des échantillons demeure totalement symétrique jusqu'à des taux de déformation pouvant atteindre $3,5 \times 10^{-2}$ suivant la température. Ces valeurs sont suffisantes pour l'étude de la limite élastique et du premier stade plastique. Au-delà, l'interprétation de l'essai de compression s'avère par essence délicate.

\subsection{DÉFormation DU RUTILE PAR GLISSEMENT SUI-} VANT LES DEUX SYSTÈMES ACTIFS. - Les premières traces de déformation plastique par glissement sont apparues à $873 \mathrm{~K}$, sans qu'aucun phénomène de maclage n'ait été observé à températures plus basses. Les différentes courbes ont été enregistrées entre $900 \mathrm{~K}$ et $1700 \mathrm{~K}$.

3.3.1 Mesure des grandeurs caractérisant la déformation. - L'aptitude des cristaux au glissement dans les deux orientations décrites précédemment a été caractérisée par l'enregistrement de courbes contrainte-déformation à différentes températures et vitesses de déformation.

L'indentation des éprouvettes dans les plaquettes d'alumine intercalées (Fig. $1 b$ ) pour éviter tout collage des échantillons sur les faces des pistons s'est avérée négligeable dans toute la gamme de ces essais. L'appareillage fournit donc tout au long d'un essai les couples 
Fig. 4a. - Axe de compression suivant [001]. Les plans de glissement actifs sont $\mathrm{P}, \mathrm{P}^{\prime}, \mathrm{P}_{\mathbf{1}}$ et $\mathrm{P}_{\mathbf{2}}$.

[Compression axis along [001]. The active slip planes are $\mathrm{P}, \mathrm{P}^{\prime}$, $\mathrm{P}_{1}$ and $\mathrm{P}_{2}$.]

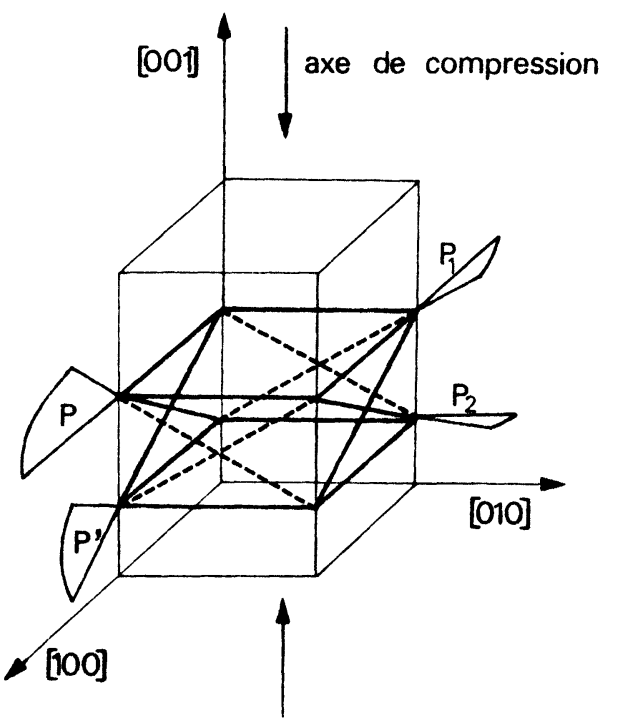

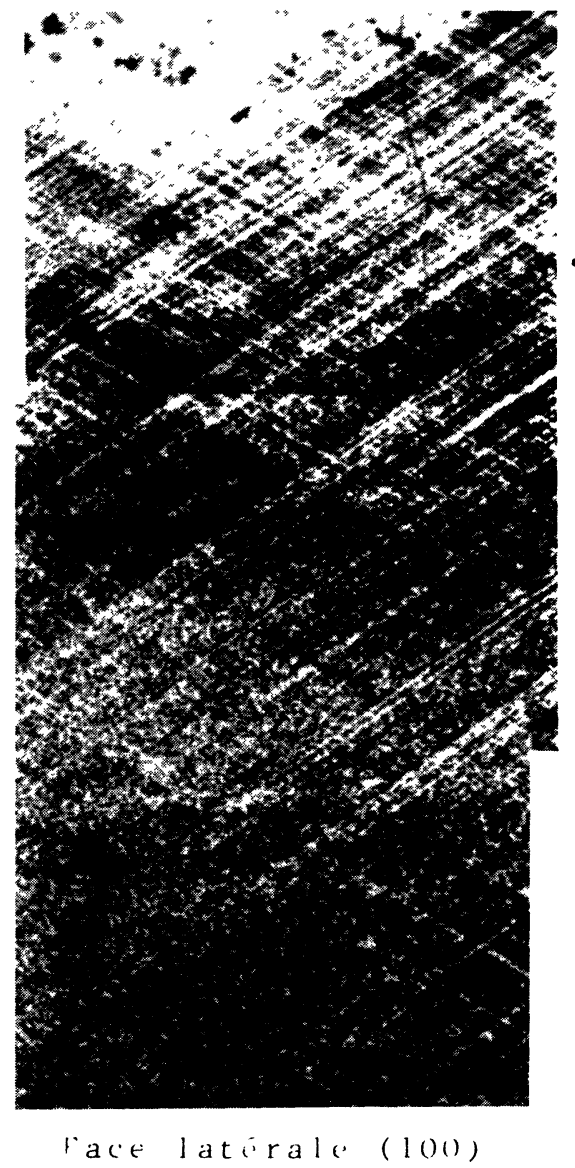

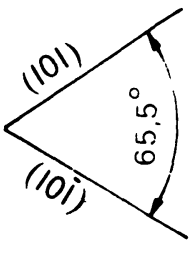

$200 \mu \mathrm{m}$

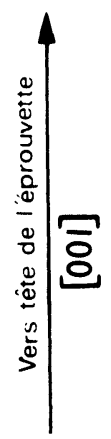

Fig. $4 b$. - Faces latérales d'une éprouvette déformée à $T=1068 \mathrm{~K}$, $\dot{\varepsilon}=1,4 \times 10^{-2}$.

[Lateral faces of a sample deformed at $T=1068 \mathrm{~K}, \dot{\varepsilon}=1.4 \times 10^{-2}$.]

de valeurs instantanées effort-allongement réel à partir desquels sont calculées les courbes rationnelles $\sigma=f(\varepsilon)$.

La majeure partie des essais a été effectuée en utilisant une vitesse de déplacement de la traverse de $20 \mu / \mathrm{mm}$. Nous avons également employé d'autres
Cône de glıssement

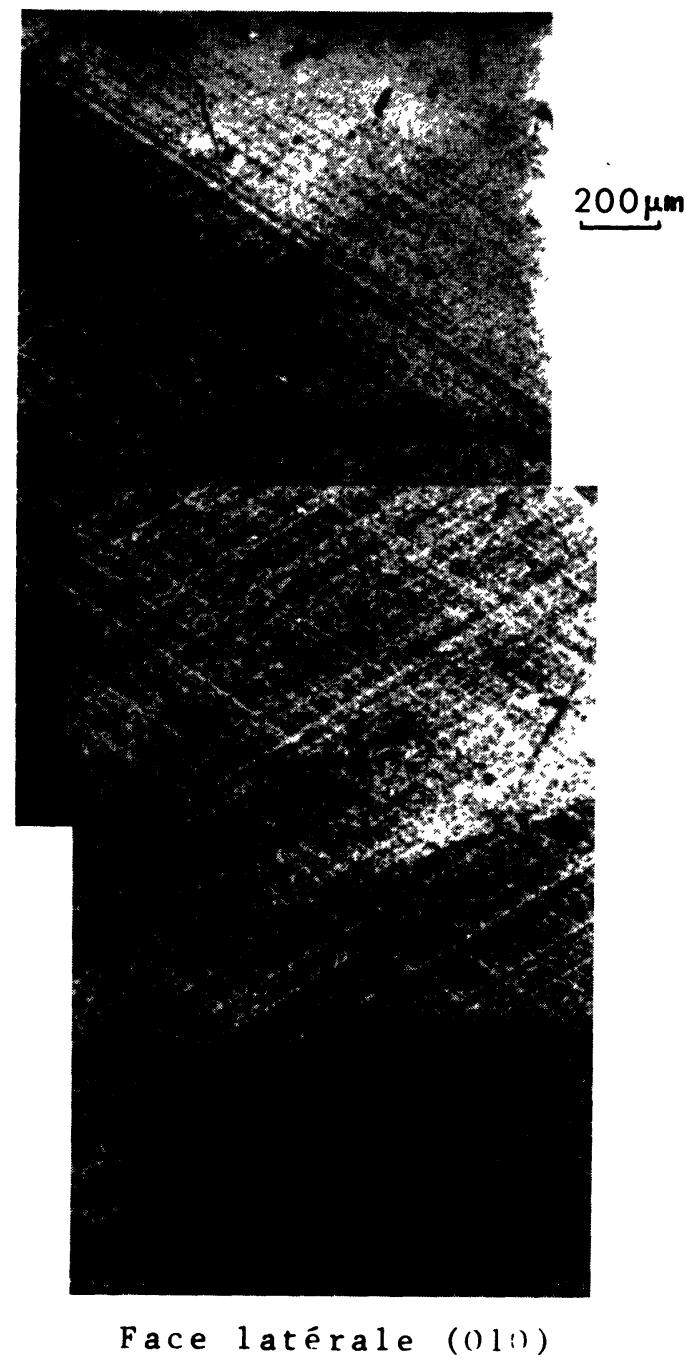

vitesses dans la gamme 5 à $200 \mu / \mathrm{mm}$. Le capteur de déplacement permet de mesurer la vitesse d'écrasement réel de l'éprouvette en fonction de sa déformation au cours d'un essai. Les enregistrements (Fig. 5, par exemple) ont montré que cette vitesse est très faible dans le domaine élastique, la majeure partie de la 


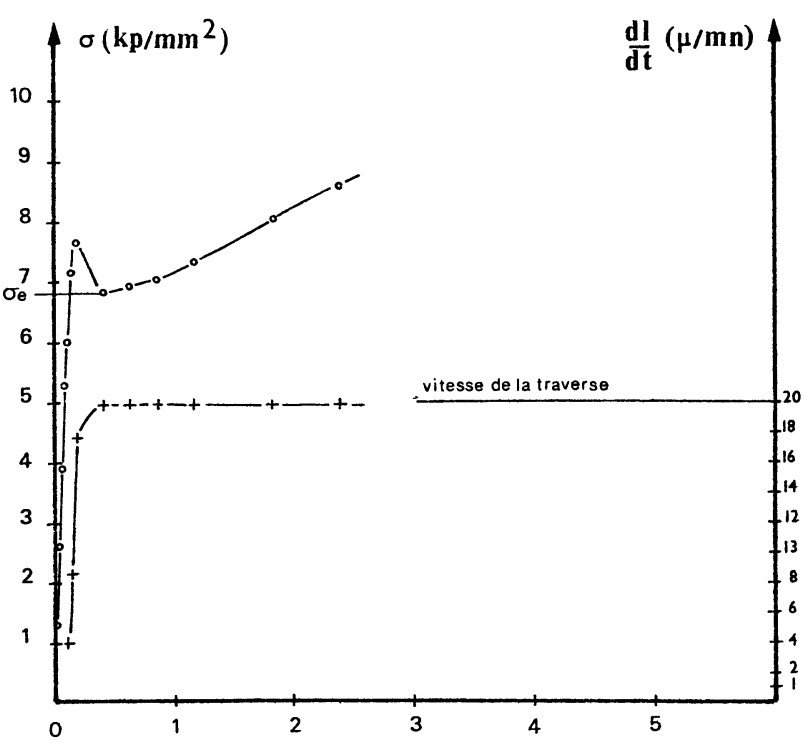

Fig. 5. - Courbe contrainte-déformation et vitesse d'écrasement réelle pour un échantillon comprimé suivant [001] $\left(T=1073 \mathrm{~K}, \dot{\varepsilon}=5,65 \times 10^{-5} \mathrm{~s}^{-1}\right)$.

[Stress-strain curve and true strain rate for a sample compressed along [001] $\left(T=1073 \mathrm{~K}, \dot{\varepsilon}=5.65 \times 10^{-5} \mathrm{~s}^{-1}\right)$.]

déformation étant alors encaissée par la machine. Elle augmente ensuite très brutalement pour atteindre la vitesse de la traverse à la limite élastique. La vitesse de déformation réelle est donc bien déterminée alors par la vitesse de la traverse et la longueur de l'échantillon, soit une vitesse $\dot{\varepsilon}$ à la limite élastique comprise entre $1,4 \times 10^{-5} \mathrm{~s}^{-1}$ et $5,65 \times 10^{-4} \mathrm{~s}^{-1}$ dans la gamme de nos essais.

3.3.2 Glissement suivant le système $\{110\}\langle 001\rangle$. - Les courbes contrainte-déformation obtenues dans cette orientation ont l'allure reproduite sur la figure 6 . Passé un niveau relativement élevé (comparativement

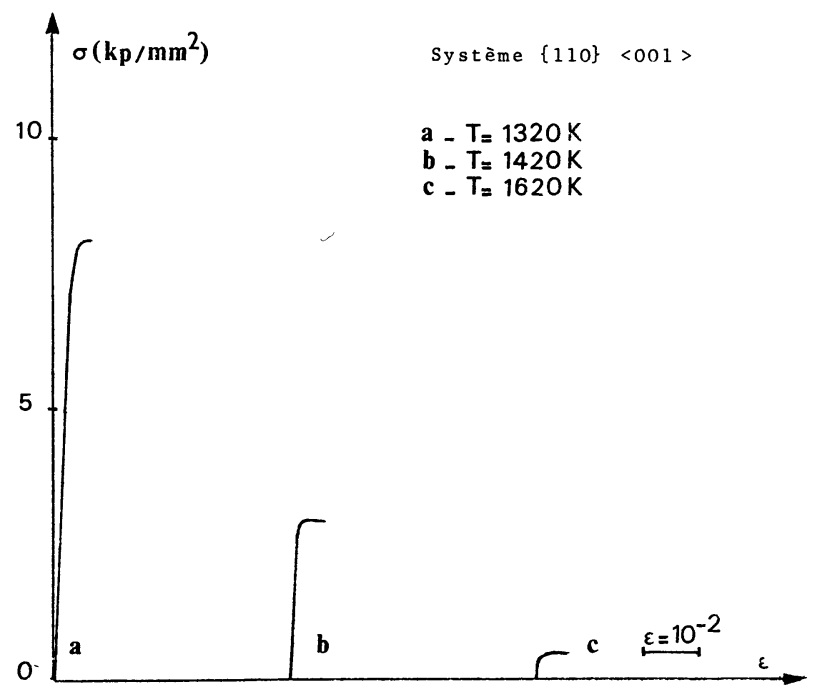

Fig. 6. - Courbes contrainte-déformation à différentes températures $\dot{\varepsilon}=5,65 \times 10^{-5} \mathrm{~s}^{-1}$ (axe de compression comme Fig. $3 a$ ).

[Stress-strain curves at different temperatures, $\dot{\varepsilon}=5.65 \times 10^{-5} \mathrm{~s}^{-1}$ (compression axis as in Fig. 3a).] à la limite élastique obtenue dans la seconde orientation aux mêmes valeurs de $T$ et $\dot{\varepsilon}$ ), la contrainte se stabilise puis décroît : une observation macroscopique révèle alors des amorces de fissures dans l'échantillon. Les observations présentées au paragraphe précédent montrent que le glissement s'amorce effectivement suivant les plans $\{110\}$ mais ne se propage pas à l'ensemble de l'échantillon.

Nous n'avons jamais réussi à obtenir une ductilité plus importante dans cette orientation même en élevant la température jusqu'à $1625 \mathrm{~K}$ et en abaissant la vitesse de déformation au maximum accessible à l'appareillage, soit $\dot{\varepsilon}=1,4 \times 10^{-5} \mathrm{~s}^{-1}$.

3.3.3 Glissement suivant le système $\{101\}<\overline{1} 01\rangle$. - La figure 7 rassemble les courbes contrainte appliquée-déformation représentatives des différentes allures obtenues à $\dot{\varepsilon}$ données en fonction de la température.

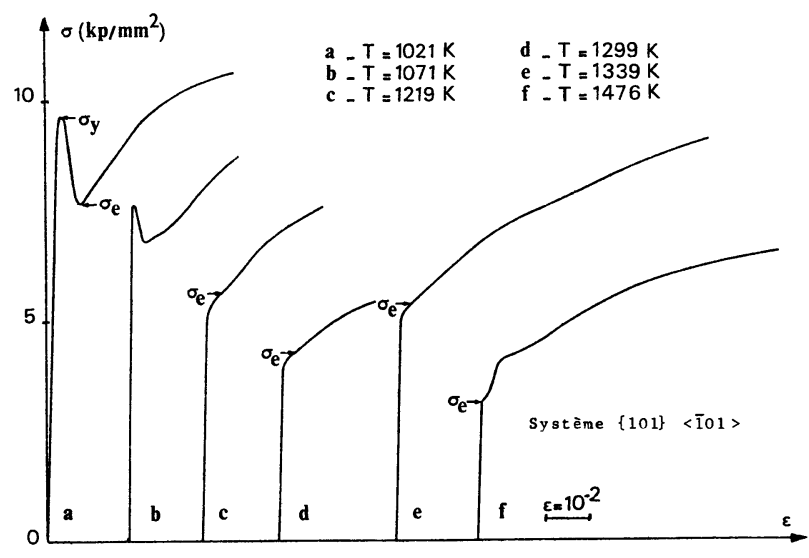

Fig. 7. - Courbes contrainte-déformation à différentes températures $\dot{\varepsilon}=5,65 \times 10^{-5} \mathrm{~s}^{-1}$ (compression suivant [001]).

[Stress-strain curves at different temperatures $\dot{\varepsilon}=5.65 \times 10^{-5} \mathrm{~s}^{-1}$ (compression along [001]).]

Immédiatement après la limite élastique $\sigma_{\mathrm{e}}$, le domaine plastique comporte une région linéaire dont la pente définit le taux de consolidation lié au glissement quadruple. On observe ensuite une rupture de pente plus ou moins progressive due au développement d'un glissement asymétrique (deux puis un plan de glissement seulement) qui survient pour de faibles déformations plastiques à températures élevées (Fig. $7 f$ ). Vers $1173 \mathrm{~K}$, le crochet de limite élastique bien marqué aux plus basses températures disparaît au profit d'un épaulement plus ou moins atténué : la limite élastique $\sigma_{\mathrm{e}}$ est alors définie comme une contrainte d'écoulement prise au début du domaine plastique linéaire.

Les contraintes de cisaillement $\tau_{\mathrm{y}}$ correspondant à la limite élastique supérieure et $\tau_{\mathrm{e}}$ à la limite élastique inférieure ont été calculées dans le plan de glissement $\{101\}$ pour la direction de glissement $\langle\overline{101}\rangle$ (relation de Schmid et Boas). Les valeurs obtenues pour deux séries d'éprouvettes ont été portées en fonction de la température sur la figure 8 . 


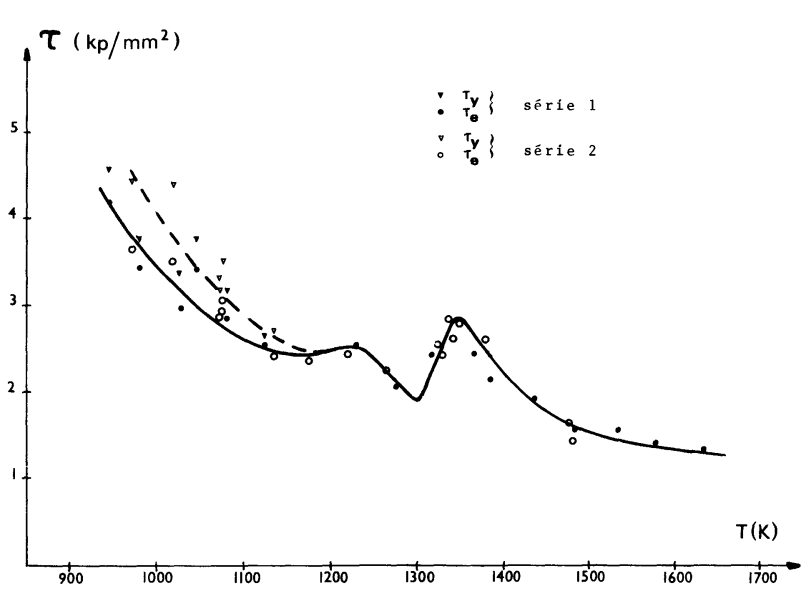

Fig. 8. - Variation des limites élastiques en fonction de la température, $\dot{\varepsilon}=5,65 \times 10^{-5} \mathrm{~s}^{-1}$ (compression suivant [001]).

[Dependance of the elastic limits on the temperature $\dot{\varepsilon}=5.65 \times 10^{-5} \mathrm{~s}^{-1}$ (compression along [001]).]

L'ensemble des points $\tau_{\mathbf{e}}$ se répartit assez bien sur une courbe unique, dont la caractéristique remarquable est, après une rapide décroissance entre 900 et $1175 \mathrm{~K}$, la présence de deux maxima à température croissante. Le premier est situé aux alentours de $1235 \mathrm{~K}$, le second, d'amplitude plus importante, vers $1345 \mathrm{~K}$. Après quoi $\tau_{\mathrm{e}}$ ne décroît plus que faiblement lorsque $T$ augmente.

4. Discussion. - 4.1 ACtivation Des SystèmeS DE GLISSEMENT. - Nos essais ont montré que pour les mêmes valeurs de la température et de la vitesse de déformation, l'activation du système $\{110\}\langle 001\rangle$ requiert une contrainte plus élevée que le système $\{101\}\langle\overline{1} 01\rangle$ et ne confère pas de ductilité au cristal. Les conclusions d'Ashbee et Smallman [7] sont similaires aux nôtres sur ce point.

L'activation plus aisée du système $\{101\}\langle\overline{1} 01\rangle$ peut surprendre $a$ priori car étant donnée la structure du rutile (Fig. 2a), 〈001 > est bien le plus court vecteur de Burgers possible pour une dislocation parfaite. Toutefois, dans un oxyde comme le rutile, les problèmes de coordination ont une grande importance; aussi, le système de glissement peut-il avant tout être conditionné par la structure de cœur des dislocations, elle-même liée à des considérations structurales. Du point de vue compacité, le squelette de la structure est constitué par le sous-réseau des anions oxygène (plus volumineux) dont l'arrangement est hexagonal compact à une légère distorsion près, le plan de base de cette structure hexagonale compacte étant le plan (100) du réseau quadratique (Fig. 2b). L'on voit que le système $\{101\}\langle\overline{1} 01\rangle$ correspond au glissement prismatique de ce réseau pseudo-hexagonal, tandis que le système $\{110\}<001>$ ne correspond à aucun glissement connu de la structure hexagonale. Or l'on sait que dans les structures hexagonales métalliques où $c^{\prime} a<1,6$, le glissement activé préfé- rentiellement est précisément le glissement prismatique [14].

De plus, comme l'ont suggéré Ashbee et Smallman [8], une dissociation des dislocations parfaites de vecteur de Burgers $\langle\overline{10} 1\rangle$ en deux partielles colinéaires ne perturbe en première approximation que le sous-réseau des cations : aussi est-elle beaucoup plus facilement envisageable qu'une dissociation du vecteur $\langle 001\rangle$, qui affecte simultanément les deux sous-réseaux [15].

4.2 COMPORTEMENT MÉCANIQUE LIÉ AU GLISSEMENT SUIVANT LE SYSTÈME $\{101\}\langle\overline{1} 01\rangle$. - L'ensemble des résultats (Fig. 7 et 8 ) obtenus pour ce système suggère fortement l'existence d'interactions dislocations glissiles-défauts ponctuels. Aux plus basses ou plus hautes tempúratures explorées, les mobilités de ces deux types de défauts sont très inégales. Aux températures intermédiaires, elles deviennent comparables, permettant ancrages et désancrages successifs des dislocations par les défauts ponctuels durant la déformation plastique elle-même. Ce mécanisme entraîne un durcissement généralisé du cristal, d'où, en particulier une remontée de la limite élastique. Il est parallèlement à l'origine de l'effet Portevin-Le Châtelier $[14,16]$ observé aux températures intermédiaires. Les enregistrements originaux des courbes effort-temps font clairement apparaître des décrochements en paliers [1]. Corrélativement, la configuration des figures de corrosion observée sur les faces d'éprouvettes déformées à ces températures est tout à fait caractéristique d'une propagation hétérogène du glissement par bandes (Fig. 9), comparée au glissement quadruple homogène observé à plus basses températures.

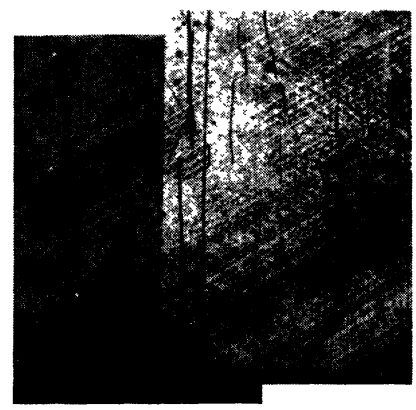

Fig. 9. - Face latérale (100) d'une éprouvette comprimée suivant [001] à $T=1317 \mathrm{~K}, \varepsilon=0,9 \times 10^{-2}$. Remarquer la répartition hétérogène des traces de glissement.

[Lateral face (100) of a sample compressed along [001] at $T=1317 \mathrm{~K}$, $\varepsilon=0.9 \times 10^{-2}$. The heterogeneous repartition of the slip can be seen.]

Les deux maxima de limite élastique successivement observés correspondent en fait à l'existence d'un double effet, que l'on peut attribuer à deux espèces ponctuelles différentes. Compte tenu de l'écart maximal à la stœchiométrie susceptible de s'instaurer à ces températures $(\S 3.1)$, ces espèces sont les impu- 
retés $\left(^{2}\right)$ majoritaires, associées ou non aux défauts de stœchiométrie proprement dits (interstitiels titane ou lacunes d'oxygène), ces derniers demeurant de toute façon minoritaires.

Pour une vitesse de déformation comparable, Ashbee et Smallman [7] ont observé un seul pic de limite élastique situé vers $1350 \mathrm{~K}$ qu'ils attribuèrent aux défauts de non-stœchiométrie seuls, les échantillons étant comprimés sous air. Cependant, les résultats obtenus par ces auteurs sur une seconde série de cristaux présumés largement sous-stochiométriques (déformation sous vide) mettent également en évidence un seul pic de limite élastique, mais situé cette fois-ci à $1273 \mathrm{~K}$, soit décalé de $75^{\circ}$ au moins vers les basses températures. Ce point nous paraît précisément remettre en cause l'argument d'Ashbee et Smallman et corroborer au contraire notre hypothèse selon laquelle, pour $p \mathrm{O}_{2}=1 \mathrm{~atm}$, le rôle prépondérant est joué par les impuretés. Dans le cas de notre étude comme en [7], l'impureté majoritaire est l'aluminium, qui semble donc à l'origine du pic communément observé vers $1350 \mathrm{~K}$; toutefois un effet moyenné sur plusieurs impuretés n'est pas à exclure. Excepté l'aluminium, la nature des autres impuretés majoritaires dans les cristaux étudiés par Ashbee et Smallman est assez différente des nôtres : ceci est susceptible d'expliquer l'absence d'un second maximum de limite élastique et les contraintes d'écoulement comparativement plus basses à partir de $1150 \mathrm{~K}$ observées par ces auteurs.

Des mesures (paramètres d'activation thermique, notamment) et des études complémentaires ont permis d'analyser de façon détaillée l'interaction dislocations-

$\left({ }^{2}\right)$ Lors des essais effectués à $T>1150 \mathrm{~K}$, on intercale entre l'éprouvette et chaque plaquette une feuille de platine qui empêche toute diffusion d'impuretés supplémentaires en provenance de l'alumine. impuretés dans le rutile stœchiométrique ; ces résultats font l'objet d'une autre publication [17].

5. Conclusion. - La conception du montage de compression présenté ici en permet une réalisation rapide et avantageuse. Cet ensemble s'est avéré capable de transmettre des forces élevées à hautes températures sous atmosphère oxydante, en conservant une rigidité et une axialité satisfaisantes. Il peut être utilisé avec d'autres atmosphères pour comprimer d'autres cristaux (en résolvant le problème d'une éventuelle réactivité de l'échantillon avec l'alumine).

Nous avons pour notre part employé cet appareillage pour déformer des monocristaux de rutile. Le balayage d'oxygène pur réalisé dans l'installation limite au maximum l'écart à la stœchiométrie susceptible de s'instaurer à hautes températures dans les monocristaux déformés. Les essais de compression effectués dans ces conditions ont permis de caractériser l'activation des systèmes de glissement dans le rutile. Le comportement mécanique des cristaux se déformant par glissement suivant le système $\{110\}\langle 001\rangle$ reste éminemment fragile. Le système $\{101\}\langle\overline{1} 01\rangle$ confère, en revanche, une ductilité notable. L'analyse des courbes contrainte-déformation correspondantes suggère l'existence d'interactions dislocations glissilesdéfauts ponctuels. Le très faible écart à la stœchiométrie sous oxygène pur conduit à invoquer ici le rôle prépondérant joué par les impuretés, que n'avaient pas mis en évidence les études antérieures.

Remerciements. - Nous remercions J. Doglioni qui a réalisé une grande partie des pièces métalliques de ce montage, ainsi que G. Guiraud et D. Guillot qui nous ont aussi apporté leur concours. Nos remerciements vont également au Professeur Fontaine, qui nous a suggéré cette étude, pour de fructueuses discussions.

\section{Bibliographie}

[1] Blanchin, M. G., Thèse Doctorat ès Sciences (1978), Lyon. [10] Baumard, J. F., Panis, D., Anthony, A. M., J. Solid State

[2] Cadoz, J. L., Castaing, J., Dolin, C., Gervais, H., PelisSIER, B., Revue Phys. Appl. 10 (1975) 247.

[3] Clavaud, B., Bull. Soc. Fr. Ceram. 109 (1975) $49 . \quad$ [12] Haul, R., Duembgen, G., J. Phys. Chem. Solids 26 (1965) 1 Chem. 20 (1977) 43

[11] Baumard, J. F., Tani, E., J. Chem. Phys. 67 (1977) 857.

[4] Pascoe, R. T., Radford, K. C., Rawling, R. D., Newey, C. [13] Blanchin, M. G., Fontaine, G., Phys. Status Solidi (a) W. A., J. Sci. Instrum. 44 (1967) 366.

[5] Moise, A., Chim. Actual. 78 (1974) 1541-1542.

[6] Faisant, P., Diplôme d'Etudes Supérieures (1975) Lyon. 29 (1975) 491 .

[7] Ashbee, K. H. G., Smallman, R. E., Proc. Roy. Soc. A 274 (1963) 195.

[8] Ashbee, K. H. G., Smallman, R. E., Williamson, G. K., Proc. Roy. Soc. A 276 (1964) 542.

[9] Picard, C., Gerdanian, P., J. Solid State Chem. 14 (1975) 66.

[14] JAOUL, B., Etude de la plasticité et application aux métaux (Dunod, Paris) 1965, pp. 179, 336 et 435.

[15] Hirthe, W. M., Brittain, J. O., J. Am. Ceram. Soc. 45 ( I 546.

[16] Caisso, J., Thèse Doctorat ès Sciences (1958) Poitiers.

[17] Blanchin, M. G., Kubin, L. P., Fontaine, G., à paraitre. 